\title{
Efficient solutions to hard computational problems by P systems with symport/antiport rules and membrane division
}

\author{
Bosheng Song a,b, Mario J. Pérez-Jiménez ${ }^{\mathrm{b}}$, Linqiang Pan ${ }^{\mathrm{a}}$ \\ a Key Laboratory of Image Information Processing and Intelligent Control, School of Automation, Huazhong University of Science and Technology, Wuhan 430074, Hubei, China \\ ${ }^{\mathrm{b}}$ Research Group on Natural Computing, Department of Computer Science and Artificial Intelligenc, University of Sevilla, Avda. Reina Mercedes s/n, 41012 Sevilla, Spain
}

Keywords:

Cell-like P system

Symport/Antiport rule

Membrane division

Subset Sum problem

QSAT problem

\begin{abstract}
A B S T R A C T
P systems are computing models inspired by some basic features of biological membranes. In this work membrane division, which provides a way to obtain an exponential workspace in linear time, is introduced into (cell-like) P systems with communication (symport/antiport) rules, where objects are never modified but they just change their places. The computational efficiency of this kind of P systems is studied. Specifically, we present a (uniform) linear time solution to the NP-complete problem, Subset Sum by using division rules for elementary membranes and communication rules of length at most 3 . We further prove that such P system allowing division rules for non-elementary membranes can efficiently solve the PSPACE-complete problem, QSAT in a uniform way.
\end{abstract}

1. Introduction

Membrane computing is a flexible and versatile branch of natural computing, which arises as an abstraction of the compartmentalized structure of living cells, and the way biochemical substances are processed in (or moved between) membrane bounded regions Păun (2000). Membrane computing is a paradigm providing computing devices called $P$ systems, which are parallel, non-deterministic and distributed computational models. Inspired by that structure, two main classes of $P$ systems have been investigated: (a) a hierarchical arrangement of membranes as in a cell Păun (2000), processing information by multisets of symbols; and (b) a net of processor units placed in the nodes of a directed graph, inspired by the cell intercommunication in tissues Martín-Vide et al. (2003) or inspired by the way neurons communicate with each other by means of short electrical impulses, identical in shape (voltage), but emitted at precise moments of time Ionescu et al. (2006). These models have two interesting properties: the capability to solve as many problems as a Turing machine (computational completeness) and the ability to solve computationally hard problems in a feasible time, by trading space for time (computational efficiency). General information on membrane computing can be found in Păun et al. (2010), Frisco (2009), and for the most up-to-date references, one can refer to the P systems website http://ppage.psystems.eu.

A basic $P$ system is based on a cell-like arrangement of membranes, that are placed in a nested hierarchical structure; each membrane delimits a compartment (also called region) where multisets of objects and rules for evolving these objects are placed. A membrane with no compartments inside is called elementary, otherwise it is called non-elementary. The outmost membrane is called a skin membrane, the space outside the skin membrane is called the environment. There are two main types of evolution rules of cell-like P systems associated with membranes: multiset rewriting rules and communication (symport/antiport) rules. The present work focuses on a class of P systems with symport/antiport rules, which were proposed in Păun and Păun (2002). Symport rules are of form $(u, i n)$ or $(u, o u t)$, and move objects of multiset $u$ through a membrane; antiport rules are of form ( $u$, out; $v$, in), and move the objects of multiset $u$ outside a membrane while moving the objects of multiset $v$ inside.

Cell-like P systems have been studied widely. Up to now, researchers have proposed lots of classes of cell-like P systems, and many of them have been proved to be computationally complete (see, e.g., Alhazov and Freund (2005), Alhazov et al. (2006), 
Bernardini and Gheorghe (2003), Ciobanu et al. (2007), Păun and Păun (2002), Păun et al. (2005)). The computational efficiency of cell-like P systems has also been investigated Păun et al. (2010). A particularly interesting class of cell-like $\mathrm{P}$ systems is that of $\mathrm{P}$ systems with active membranes Păun (2000), which contain object evolution rules, in communication rules, out communication rules, dissolving rules and membrane division rules. Membrane division rules can generate an exponential workspace in polynomial time (even in linear time). Therefore, $P$ systems with active membranes can be used to solve computationally hard problems by a time-space trade-off Păun (2001), Pérez-Jiménez and Riscos-Núñez (2004), Pérez-Jiménez and Riscos-Núñez (2005).

In this work, membrane division is introduced into cell-like $\mathrm{P}$ systems, and they evolve by means of symport/antiport or division rules, that is, we present a class of $P$ systems with symport/antiport rules and membrane division, where objects are never modified but they just change their places, moreover, objects can be communicated with the environment only through the skin membrane. The computational efficiency of such kind of P systems is studied. Specifically, we present polynomial time solutions to the subset Sum problem by using division rules for elementary membranes and for the QSAT problem by using division rules for non-elementary membranes. In both cases, the solutions are uniform and they are obtained by using communication rules of length at most 3 .

\section{Preliminaries}

In this Section, we only introduce some basic notions and notations from formal languages theory. Readers can refer to Rozenberg and Salomaa (1997) for details.

An alphabet $\Sigma$ is a non-empty set and their elements are called symbols. An ordered finite sequence of symbols over $\Sigma$ forms a string. The length of a string $u$, denoted by $|u|$, is the number of occurrences of symbols it contains. We denote by $\Sigma^{*}$ the set of all strings over $\Sigma$. The empty string (with length 0 ) is denoted by $\lambda$, and by $\Sigma^{+}=\Sigma^{*} \backslash\{\lambda\}$ we denote the set of non-empty strings.

For an alphabet $\Sigma$, a multiset over $\Sigma$ is a pair $(\Sigma, f)$ where $f: \Sigma \rightarrow \mathbb{N}$ is a mapping, $\mathbb{N}$ is the set of natural numbers. If $m=(\Sigma, f)$ is a multiset, then its support is defined as $\operatorname{supp}(m)=\{x \in \Sigma \mid f(x)>0\}$. If $\operatorname{supp}(m)=\left\{a_{1}, \ldots, a_{k}\right\}$ then we denote $m=\left\{a_{1}^{f\left(a_{1}\right)}, \ldots, a_{k}^{f\left(a_{k}\right)}\right\}$. A multiset is finite if its support is a finite set. We denote by $\emptyset$ the empty multiset and by $M_{f}(\Sigma)$ the set of all finite multisets over $\Sigma$. If $m_{1}=\left(\Sigma, f_{1}\right), m_{2}=\left(\Sigma, f_{2}\right)$ are multisets over $\Sigma$, then the union of $m_{1}$ and $m_{2}$, denoted by $m_{1}+m_{2}$, is the multiset $(\Sigma, g)$, where $g(x)=f_{1}(x)+f_{2}(x)$ for each $x \in \Sigma$; the relative complement of $m_{2}$ in $m_{1}$, denoted by $m_{1} \backslash m_{2}$, is the multiset $(\Sigma, g)$, where $g(x)=f_{1}(x)-f_{2}(x)$ if $f_{1}(x) \geq f_{2}(x)$, and $g(x)=0$ otherwise.

\section{P systems with symport/antiport rules and membrane division}

In this Section, we study P systems with symport/antiport rules, a class of computing devices aiming to abstract the active transport of molecules across the membranes. In this kind of P systems, objects are processed in a pure communicative way, that is, objects do not evolve and only change their places during the computation process. Membrane division rules are allowed in such P systems.

Definition 1. A P system with symport/antiport rules and membrane division of degree $q \geq 1$ is a tuple $\Pi=$ $\left(\Gamma, \mathcal{E}, \mu, \mathcal{M}_{1}, \ldots, \mathcal{M}_{q}, \mathcal{R}_{1}, \ldots, \mathcal{R}_{q}, i_{\text {out }}\right)$, where:

- $\Gamma$ is an alphabet (the working alphabet) and $\mathcal{E} \subseteq \Gamma$;

- $\mu$ is a rooted tree with $q$ nodes labeled by $1, \ldots, q$;

- $\mathcal{M}_{i}, 1 \leq i \leq q$, are finite multisets over $\Gamma$;

- $\mathcal{R}_{i}, 1 \leq i \leq q$, are finite sets of rules of the following forms: $\left(a_{1}\right)$ Symport rules: $(u$, out $)$ or $(u$, in $)$, for $u \in M_{f}(\Gamma),|u|>0$;

$\left(a_{2}\right)$ Antiport rules: $\left(u\right.$, out; $v$, in), for $u, v \in M_{f}(\Gamma),|u|>0,|v|>0$;

(b) Division rules: $[a]_{i} \rightarrow[b]_{i}[c]_{i}$, for $i \in\{2, \ldots, q\}, i \neq i_{\text {out }}, a, b$, $c \in \Gamma$;

- $i_{\text {out }} \in\{0,1, \ldots, q\}$.

A P system with symport/antiport rules and membrane division of degree $q \geq 1$ can be viewed as a set of $q$ membranes labeled by $1, \ldots, q$, arranged in a hierarchical structure of a rooted tree (the root labeled by 1 is called the skin membrane), such that: (a) $\mathcal{M}_{1}, \ldots, \mathcal{M}_{q}$ represent the finite multisets of objects initially placed in the $q$ membranes of the system; (b) $\mathcal{E}$ is the set of objects initially located in the environment of the system, all of them available in an arbitrary number of copies; (c) $\mathcal{R}_{1}, \ldots, \mathcal{R}_{q}$ are finite sets of rules ( $\mathcal{R}_{i}$ corresponds to the membrane $i$ of $\mu$ ); (d) $i_{\text {out }}$ is a distinguished region which will encode the output of the system. We use the term region $i(0 \leq i \leq q)$ to refer to membrane $i$ in case $1 \leq i \leq q$ and to refer to the environment in case $i=0$. The length of a symport rule $(u, o u t)$ or ( $u$, in) (an antiport rule ( $u$, out; $v$, in $)$, respectively) is defined as $|u|(|u|+|v|$, respectively).

For each membrane $i \in\{2, \ldots, q\}$, we denote by $p(i)$ the parent of membrane $i$ in the rooted tree $\mu$, the "parent" of the skin membrane is the environment, denoted by $p(1)=0$.

A configuration of such a P system at any moment is described by the current membrane structure (the rooted tree), together with all multisets of objects over $\Gamma$ associated with the regions of this membrane structure and the multiset of objects over $\Gamma \backslash \mathcal{E}$ associated with the environment at that moment. The initial configuration of $\left(\Gamma, \mathcal{E}, \mu, \mathcal{M}_{1}, \ldots, \mathcal{M}_{q}, \mathcal{R}_{1}, \ldots, \mathcal{R}_{q}, i_{\text {out }}\right)$ is $\left(\mu, \mathcal{M}_{1}, \ldots, \mathcal{M}_{q} ; \emptyset\right)$.

A symport rule $(u$, out $) \in \mathcal{R}_{i}$ is applicable to a configuration at a moment if membrane $i$ contains multiset $u$ at that moment. When such a rule is applied, multiset $u$ is sent to the parent of membrane $i$. A symport rule $(u, i n) \in \mathcal{R}_{i}$ is applicable to a configuration at a moment if the parent of membrane $i$ contains multiset $u$ at that moment. When such a rule is applied, multiset $u$ enters membrane $i$ from the parent of $i$.

An antiport rule $(u$, out; $v$, in $) \in \mathcal{R}_{i}$ is applicable to a configuration at moment if membrane $i$ contains multiset $u$ and its parent contains multiset $v$ at that moment. When such a rule is applied, multiset $u$ from membrane $i$ is sent out of membrane $i$, and simultaneously, multiset $v$ enters region $i$ from the parent of $i$.

A division rule $[a]_{i} \rightarrow[b]_{i}[c]_{i}$ is applicable to a configuration at a moment if the following conditions hold at that moment: (1) membrane $i$ contains object $a$; (2) membrane $i$ is neither the skin membrane nor the output membrane. When applying such a rule, membrane $i$ is divided into two membranes with the same label: in the first copy, object $a$ is replaced by object $b$, in the second one object $a$ is replaced by object $c$, and all the objects in the original membrane, different from the object triggering the rule, are replicated in the two new membranes. Besides, if membrane $i$ is a non-elementary membrane, then all membranes inside membrane $i$ as well as objects contained in them, will be replicated in each of the new membranes.

The rules of a P system with symport/antiport rules and membrane division are used in a maximally parallel way. At each step, a maximal multiset of rules is applied (no further rule can be added being applicable) with the following restriction: when a membrane is divided, the division rule is the only one which is applied for that membrane at that step, the objects inside that membrane do not evolve by means of communication rules. The objects in the new membranes resulting from division could participate in the interaction with the objects in the (upper or lower) neighbor of membranes by means of communication rules at the next step if these new membranes are not divided once again.

Starting from the initial configuration and applying rules as described above, one obtains a sequence of consecutive 
configurations. Each passage from a configuration $\mathcal{C}$ to a next configuration $\mathcal{C}^{\prime}$ is called a transition and denoted by $\mathcal{C} \Rightarrow \mathcal{C}^{\prime}$. A configuration is a halting configuration if no rule of the system is applicable to it. A computation of such a P system is a (finite or infinite) sequence of transitions between configurations such that: (1) the first term of the sequence is the initial configuration; (2) each non-first term of the sequence is obtained from the previous configuration by applying rules in a maximally parallel way with the above mentioned restriction; (3) if the sequence is finite, then the last term of the sequence is a halting configuration, and such a computation is called a halting computation. Only halting computations give a result, encoded by the multiset of objects present in the output region $i_{\text {out }}$.

\subsection{Recognizer P systems with symport/antiport rules and membrane division}

Recognizer P systems were introduced in Pérez-Jiménez et al. (2006), as a natural framework to solve decision problems.

Definition 2. A recognizer P system with symport/antiport rules and membrane division of degree $q \geq 1$ is a tuple

$\Pi=\left(\Gamma, \mathcal{E}, \Sigma, \mu, \mathcal{M}_{1}, \ldots, \mathcal{M}_{q}, \mathcal{R}_{1}, \ldots, \mathcal{R}_{q}, i_{\text {in }}, i_{\text {out }}\right)$,

such that:

- $\left(\Gamma, \mathcal{E}, \mu, \mathcal{M}_{1}, \ldots, \mathcal{M}_{q}, \mathcal{R}_{1}, \ldots, \mathcal{R}_{q}, i_{\text {out }}\right)$ is a P system with symport/antiport rules and membrane division of degree $q \geq 1$;

- $\Gamma$ has two distinguished objects yes and no, with at least one copy of them present in some multisets $\mathcal{M}_{1}, \ldots, \mathcal{M}_{q}$, but none of them present in $\mathcal{E}$;

- $\Sigma$ is an (input) alphabet strictly contained in $\Gamma$, and such that $\mathcal{E} \subseteq \Gamma \backslash \Sigma$;

- $\mathcal{M}_{1}, \ldots, \mathcal{M}_{q}$ are finite multisets over $\Gamma \backslash \Sigma$;

- $i_{\text {in }} \in\{1, \ldots, q\}$ is the input membrane, and $i_{\text {out }}=0$;

- all computations halt;

- if $\mathcal{C}$ is a computation of $\Pi$, then either object yes or object no (but not both) must have been released into the environment, and only at the last step of the computation.

For each finite multiset $w \in M_{f}(\Sigma)$, the computation of a P system with symport/antiport rules and membrane division with input $w$ starts from a configuration of the form $\left(\mu, \mathcal{M}_{1}, \ldots, \mathcal{M}_{i_{\text {in }}}+\right.$ $\left.w, \ldots, \mathcal{M}_{q}, \emptyset\right)$, where the input multiset $w$ is added to the contents of the input membrane $i_{\text {in }}$.

We denote by $\mathbf{C D}_{e} \mathbf{C}(k)\left(\mathbf{C D}_{n e} \mathbf{C}(k)\right.$, respectively) the class of recognizer $\mathrm{P}$ systems with division rules for elementary membranes (non-elementary membranes, respectively) whose symport/antiport rules have length at most $k$.

\subsection{Polynomial complexity classes of recognizer P systems}

Next, we define the concept of polynomial time solvability (in a uniform way) by means of family of P systems (see Pérez-Jiménez (2005) for details).

Definition 3. A decision problem $X=\left(I_{X}, \theta_{X}\right)$ is solvable in polynomial time by a family $\Pi=\{\Pi(n) \mid n \in \mathbb{N}\}$ of recognizer P systems from $\mathbf{C D}_{e} \mathbf{C}(k)$ or $\mathbf{C D}_{n e} \mathbf{C}(k)$, in a uniform way, if the following conditions hold:

1. The family $\Pi$ is polynomially uniform by Turing machines.

2. There exists a pair (cod, $s)$ of polynomial-time computable functions over $I_{X}$ such that: (a) for each instance $u \in I_{X}, s(u)$ is a natural number and $\operatorname{cod}(u)$ is an input multiset of the system $\Pi(s(u)$ ); (b) for each $n \in \mathbb{N}, s^{-1}(n)$ is a finite set; and (c) the family $\Pi$ is polynomially bounded, sound and complete with regard to $(X, \operatorname{cod}, s)$.

We denote by $\mathbf{P} \mathbf{M C}_{\mathbf{C D}_{e} \mathbf{C}(k)}$ ( $\mathbf{P} \mathbf{M} \mathbf{C}_{\mathbf{C D}_{n e} \mathbf{C}(k)}$, respectively) the set of all decision problems which can be solved in a uniform way and polynomial time by means of recognizer P systems from $\mathbf{C D}_{e} \mathbf{C}(k)$ $\left(\mathbf{C D}_{n e} \mathbf{C}(k)\right.$, respectively).

\section{Solving the Subset Sum problem by using $\mathrm{CD}_{\boldsymbol{e}} \mathrm{C}(3)$}

Subset Sum problem is a numerical NP-complete problem, which has been investigated widely in membrane computing. Specifically, different (uniform) polynomial time solutions have been provided by using families of $\mathrm{P}$ systems with active membranes Pérez-Jiménez and Riscos-Núñez (2005), P systems with membrane creation Gutiérrez-Naranjo et al. (2005), and tissue P systems with cell division Díaz-Pernil et al. (2007). Next, we present a solution to the subset sum problem by combining the symport/antiport rules used in tissue-like P systems and the membrane division of $\mathrm{P}$ systems with active membranes.

The subset sum problem is described as follows: Given a finite set $A$, a weight function, $w: A \rightarrow \mathbb{N}$, and a constant $k \in \mathbb{N}$, determine whether or not there exists a subset $B \subseteq A$, such that $w(B)=k$.

Let $g: \mathbb{N} \times \mathbb{N} \rightarrow \mathbb{N}$ be the function defined by $g(n$, $k)=((n+k)(n+k+1) / 2)+n$. It is a primitive recursive and bijective function. We denote $g(n, k)=\langle n, k\rangle$.

For each $(n, k) \in \mathbb{N} \times \mathbb{N}$, we consider the recognizer P system from $\mathbf{C D}_{e} \mathbf{C}(3)$

$\Pi(\langle n, k\rangle)=\left(\Gamma, \mathcal{E}, \Sigma, \mu, \mathcal{M}_{1}, \mathcal{M}_{2}, \mathcal{R}_{1}, \mathcal{R}_{2}, i_{\text {in }}, i_{\text {out }}\right)$,

defined as follows:

- $\Sigma=\{q\} \cup\left\{v_{i} \mid 1 \leq i \leq n\right\}$

- $\mathcal{E}=\left\{\alpha_{i} \mid 1 \leq i \leq n+\lceil\log n\rceil+\lceil\log (k+1)\rceil+7\right\} \cup\left\{a_{i} \mid 1 \leq i \leq\right.$

$n+\lceil\log n\rceil+\lceil\log (k+1)\rceil+1\} \cup\left\{b_{i} \mid 1 \leq i \leq n+\lceil\log n\rceil+\lceil\log (k+\right.$

$1)\rceil+2\} \cup\left\{c_{i} \mid 1 \leq i \leq n+\lceil\log n\rceil+\lceil\log (k+1)\rceil+4\right\} \cup\left\{d_{i, j, l} \mid 1 \leq\right.$

$i \leq n, 1 \leq j \leq\lceil\log (k+1)\rceil, 1 \leq l \leq n+j+1\} \cup\{e, p\}$;

- $\Gamma=\Sigma \cup \mathcal{E} \cup\left\{A_{i}, B_{i} \mid 1 \leq i \leq n\right\} \cup\{g, h, m, n, z$, yes, no $\}$;

- $\mu=\left[[]_{2}\right]_{1}$;

- $\mathcal{M}_{1}=\left\{d_{1,1,1}, \ldots, d_{1,\lceil\log (k+1)\rceil, 1}, d_{2,1,1}, \ldots, d_{2,\lceil\log (k+1)\rceil, 1}, \ldots\right.$, $\left.d_{n, 1,1}, \ldots, \quad d_{n,[\log (k+1)\rceil, 1}, \quad a_{1}, \quad b_{1}, \quad c_{1}, \quad n, \quad \alpha_{1}, \quad y e s, n o\right\} ;$ and $\mathcal{M}_{2}=\left\{g, h, m, A_{1}, \ldots, A_{n}\right\}$

- $i_{\text {in }}=2$ istheinputmembrane; and $i_{\text {out }}=0$ istheoutputregion;

- The set $\mathcal{R}_{1}$ consists of the following rules:

$r_{1, i} \equiv\left(\alpha_{i}\right.$, out $; \alpha_{i+1}$, in $), 1 \leq i \leq n+\lceil\log n\rceil+\lceil\log (k+1)\rceil+6$.

$r_{2, i} \equiv\left(a_{i}\right.$, out $; a_{i+1}^{2}$, in $), 1 \leq i \leq n+\lceil\log n\rceil+\lceil\log (k+1)\rceil$.

$r_{3, i} \equiv\left(b_{i}\right.$, out $; b_{i+1}^{2}$, in $), 1 \leq i \leq n$.

$r_{4, i} \equiv\left(b_{i}\right.$, out $; b_{i+1}$, in $), n+1 \leq i \leq n+\lceil\log n\rceil+\lceil\log (k+1)\rceil+1$.

$r_{5, i} \equiv\left(c_{i}\right.$, out $; c_{i+1}^{2}$, in $), 1 \leq i \leq n$.

$r_{6, i} \equiv\left(c_{i}\right.$, out $; c_{i+1}$, in $), n+1 \leq i \leq n+\lceil\log n\rceil+\lceil\log (k+1)\rceil+3$

$r_{7, i, j, l} \equiv\left(d_{i, j, l}\right.$, out $; d_{i, j, l+1}^{2}$, in $)\left\{\begin{array}{l}1 \leq i \leq n, \quad 1 \leq j \leq\lceil\log (k+1)\rceil \\ 1 \leq l \leq n+j\end{array}\right.$

$r_{8} \equiv\left(a_{n+\lceil\log n\rceil+\lceil\log (k+1)\rceil+1}\right.$, out; $p$, in $)$.

$r_{9} \equiv\left(b_{n+\lceil\log n\rceil+\lceil\log (k+1)\rceil+2}\right.$, out; e, in $)$.

$r_{10} \equiv(m$ nyes, out $)$.

$r_{11} \equiv\left(n \alpha_{n+\lceil\log n\rceil+\lceil\log (k+1)\rceil+7} n o\right.$, out $)$.

- The set $\mathcal{R}_{2}$ consists of the following rules:

$r_{12, i} \equiv\left[A_{i}\right]_{2} \rightarrow\left[B_{i}\right]_{2}[z]_{2}, 1 \leq i \leq n$.

$r_{13, i} \equiv\left(B_{i}\right.$, out $; d_{i, 1, n+2}^{2}$, in $), 1 \leq i \leq n$.

$r_{14, i, j, l} \equiv\left(d_{i, j, l}\right.$, out $; d_{i, j+1, l+1}^{2}$, in $)\left\{\begin{array}{l}1 \leq i \leq n, \quad 1 \leq j \leq\lceil\log (k+1)\rceil-1 \\ n+2 \leq l \leq n+\lceil\log (k+1)\rceil\end{array}\right.$

$r_{15, i} \equiv\left(d_{i,\lceil\log (k+1)\rceil, n+\lceil\log (k+1)\rceil+1} v_{i}\right.$, out; $p$, in $), 1 \leq i \leq n$.

$r_{16} \equiv(p q$, out $)$.

$r_{17} \equiv(g$, out $; e$, in $)$. 
$r_{18} \equiv(e p, o u t)$

$r_{19} \equiv(e q$, out $)$.

$r_{20} \equiv\left(h\right.$, out $; c_{n+\lceil\log n\rceil+\lceil\log (k+1)\rceil+4}$, in $)$.

$r_{21} \equiv\left(e m c_{n+\lceil\log n\rceil+\lceil\log (k+1)\rceil+4}\right.$, out $)$.

The computation process can be divided in the following phases.

- Generation phase: at the first $n$ steps, all possible subsets of $A$ are generated by applying membrane division rules to membranes with label 2. Simultaneously, $2^{n}$ copies of objects $d_{i, j, n+1}, a_{n+1}, b_{n+1}$, $c_{n+1}$ are produced in membrane 1 , and the counter $\alpha_{i}$ evolves in membrane 1.

- Pre-checking phase: after the generation phase, there are $2^{n}$ copies of membrane 2 , each of them containing a subset of $A$. Then as many copies of object $p$ as the weight of the corresponding subset are introduced in membrane 2 . To this aim, we should introduce enough copies of $a_{i}$ in membrane 1 first, then as many copies of $p$ as object $a_{n+[\log n\rceil+[\log (k+1)\rceil+1}$ will be introduced in membrane 1 .

- Checking phase: in each membrane with label 2, the number of copies of objects $p$ and $q$ are compared by sending all possible pairs $(p, q)$ to membrane 1 . After doing that, a membrane with label 2 will encode a subset of $A$ whose weight is equal to $k$ if and only if no object $p$ or $q$ remains in that membrane.

- Output phase: after the checking phase, the system sends an affirmative answer to the environment if there exists at least one membrane with label 2 without objects $p$ or $q$; or a negative answer if each membrane with label 2 contains some object(s) $p$ or $q$.

\subsection{An overview of the computation}

First, we consider a pair (cod, s) of polynomial time functions over the set of instances of the subset sum problem. For that, let $u=(A, w, k)$ be an instance of the problem and $A=\left\{z_{1}, z_{2}, \ldots, z_{n}\right\}$. Then,

- $\operatorname{cod}(u)=\left\{v_{i}^{j} \mid w\left(z_{i}\right)=j \wedge 1 \leq i \leq n\right\} \cup\left\{q^{k}\right\}$, where $v_{i}^{j}$ ( $j$ copies of object $v_{i}$ ) represents that $j$ is the weight of element $z_{i}$.

- $s(u)=\langle n, k\rangle$.

In this situation, instance $u=(A, w, k)$ will be processed by system $\Pi(s(u))$ with input multiset $\operatorname{cod}(u)$. In what follows, we describe informally how the recognizing $\mathrm{P}$ system $\Pi(s(u))$ from $\mathbf{C D}_{e} \mathbf{C}(3)$ with input $\operatorname{cod}(u)$ works.

At the initial configuration, we have objects $a_{1}, b_{1}, c_{1}, n, \alpha_{1}, d_{1,1,1}$, $\ldots, d_{1,[\log (k+1)\rceil, 1}, d_{2,1,1}, \ldots, d_{2,[\log (k+1)\rceil, 1}, \ldots, d_{n, 1,1}, \ldots, d_{n,\lceil\log (k+1)\rceil, 1}$, yes, no in membrane 1 , objects $A_{1}, \ldots, A_{n}, g, h, m, \operatorname{cod}(u)$ in membrane 2 .

The generation phase spends $n$ steps and the division rules $r_{12, i}$ are applied in membrane 2 producing $2^{n}$ copies of membrane 2 , each of them contains a subset of $A$; simultaneously, in membrane 1 , by using rules $r_{2, i}, r_{3, i}, r_{5, i}, r_{7, i, j, l}$, objects $a_{i}, b_{i}, c_{i}, d_{i, j, l}$ will be duplicated until getting $2^{n}$ copies in exactly $n$ steps; besides, by applying rule $r_{1, i}$, the counter object $\alpha_{i}$ increases its subscript by 1 at each step. Rule $r_{1, i}$ will be applied in the whole computation process except for the last step in the case that the answer is negative. Note that object $z$ is an idle object in cells with label 2 .

The pre-checking phase starts at step $n+1$. In this phase, we should introduce enough copies of object $p$ in each membrane 2 , but those objects are first introduced in membrane 1. Specifically, at step $n+1,2^{n+1}$ copies of objects $d_{i, j, n+2}$ are produced in membrane 1 by applying rules $r_{7, i, j, n+1}(1 \leq i \leq n, 1 \leq j \leq\lceil\log (k+1)\rceil)$. At next step, by using rules $r_{13, i}$, one copy of object $B_{i}$ in each membrane 2 is exchanged with two copies of object $d_{i, 1, n+2}$ in membrane 1 (at that step, in all membranes 2 there are at most $2^{n}$ copies of object $B_{i}$, and in membrane 1 , there are $2^{n+1}$ copies of object $d_{i, 1, n+2}$ ).
Besides, at step $n+2$, objects $d_{i, j, n+2}(1 \leq i \leq n, 2 \leq j \leq\lceil\log (k+1)\rceil)$ are duplicated by using rules $r_{7, i, n+2}$, and $2^{n+2}$ copies of objects $d_{i, j, n+3}$ are produced in membrane 1 . At step $n+3$, objects $d_{i, j, n+3}$ $(1 \leq i \leq n, 3 \leq j \leq\lceil\log (k+1)\rceil)$ are duplicated by using rules $r_{7, i j, n+3}$, and $2^{n+3}$ copies of objects $d_{i, j, n+4}$ are produced in membrane 1 . Simultaneously, each copy of object $d_{i, 1, n+2}$ in all membranes 2 is exchanged with two copies of object $d_{i, 2, n+3}$ in membrane 1 (at that step, in all membranes 2 there are at most $2^{n+1}$ copies of object $d_{i, 1, n+2}$, and in membrane 1 , there are $2^{n+2}$ copies of object $d_{i, 2, n+3}$ ). By applying rules $r_{7, i, j, l}$ and $r_{14, i, j, l}$ as many times as possible, at step $n+\lceil\log (k+1)\rceil+1$, in each membrane 2 that contains object $B_{i}$, we obtain $2^{\lceil\log (k+1)\rceil}$ copies of object $d_{i,[\log (k+1)\rceil, n+\lceil\log (k+1)\rceil+1}$, so at least $k+1$ copies will be available.

From step $n+1$ to step $n+\lceil\log n\rceil+\lceil\log (k+1)\rceil$, object $a_{i}$ will be duplicated by using rule $r_{2, i}$, that is, at step $n+\lceil\log n\rceil+\lceil\log (k+1)\rceil$, there are $2^{n+\lceil\log n\rceil+\lceil\log (k+1)\rceil}$ copies of object $a_{n+\lceil\log n\rceil+\lceil\log (k+1)\rceil+1}$ in membrane 1 . In this process, counters $b_{i}, c_{i}$ increase their subscripts by applying $r_{4, i}, r_{6, i}$.

At step $n+\lceil\log n\rceil+\lceil\log (k+1)\rceil+1$, object $a_{n+\lceil\log n\rceil+\lceil\log (k+1)\rceil+1}$ in membrane 1 is exchanged with object $p$ from the environment by using rule $r_{8}$. All copies of objects $b_{i}, c_{i}$ increase their subscripts at this step.

At step $n+\lceil\log n\rceil+\lceil\log (k+1)\rceil+2$, object $b_{n+\lceil\log n\rceil+\lceil\log (k+1)\rceil+2}$ has $2^{n}$ copies in membrane 1 and each such copy is exchanged with object $e$ from the environment (rule $r_{9}$ ). By using rule $r_{6, i}, 2^{n}$ copies of object $c_{n+\lceil\log n\rceil+\lceil\log (k+1)\rceil+3}$ will present in membrane 1 . Simultaneously, by applying rules $r_{15, i}$ in all membranes 2 , for each element $B_{i}$ in the subset associated with the membrane we get $\min \left\{2^{\lceil\log (k+1)\rceil}, w\left(s_{i}\right)\right\}$ copies of object $p$.

The checking phase starts at step $n+\lceil\log n\rceil+\lceil\log (k+1)\rceil+3$. In this step, by using rule $r_{16}$, all pairs of objects $p$ and $q$ present in any membrane with label 2 are sent to membrane 1 . In this way, if the weight of the subset associated with a membrane 2 is equal to $k$, then no object $p$ or $q$ remains in this membrane at the next step. Otherwise, at least one copy of object $p$ or $q$ will remain in the membrane. Simultaneously, in every membrane 2 object $g$ is exchanged with object $e$ in membrane 1 (rule $r_{17}$ ), that is, each membrane 2 will contain one copy of object $e$; object $c_{i}$ increases its subscript, and $2^{n}$ copies of object $c_{n+\lceil\log n\rceil+\lceil\log (k+1)\rceil+4}$ appear in membrane 1 .

When the checking phase finishes, the output phase starts at step $n+\lceil\log n\rceil+\lceil\log (k+1)\rceil+4$. There are two cases.

- There exists at least one membrane 2 with a subset whose weight is equal to $k$. In this case, at step $n+\lceil\log n\rceil+\lceil\log (k+1)\rceil+4$, if a membrane 2 contains at least one copy of object $p$ or $q$, then object $e$ will be sent to membrane 1 by using rule $r_{18}$ or $r_{19}$; otherwise, object $e$ will remain in that membrane. Simultaneously, object $h$ in all membranes 2 is exchanged with object $c_{n+[\log n\rceil+\lceil\log (k+1)\rceil+4}$ by using rule $r_{20}$ (each membrane 2 will contain one copy of object $\left.c_{n+\lceil\log \eta\rceil+\lceil\log (k+1)\rceil+4}\right)$. At the next step, by applying rule $r_{21}$, objects $e, m, c_{n+\lceil\log \eta\rceil+\lceil\log (k+1)\rceil+4}$ are sent to membrane 1 in case the weight of the subset in that membrane 2 is equal to $k$. At step $n+\lceil\log n\rceil+\lceil\log (k+1)\rceil+6$, by using rule $r_{10}$, objects $m$, $n$, yes are sent to the environment and the computation halts. Thus, the answer of the system is affirmative.

- There is no membrane 2 with a subset whose weight is equal to $k$. In this case, at step $n+\lceil\log n\rceil+\lceil\log (k+1)\rceil+4$, object $e$ is sent to membrane 1 from each membranes 2 by using rule $r_{18}$ or $r_{19}$. Simultaneously, by applying rule $r_{20}$, each membrane 2 will contain one copy of object $c_{n+\lceil\log n\rceil+\lceil\log (k+1)\rceil+4}$. At the next two steps, only the counter object $\alpha_{i}$ evolves by applying rule $r_{1, i}$. At step $n+\lceil\log n\rceil+\lceil\log (k+1)\rceil+7$, by using rule $r_{11}$, objects $n, \alpha_{n+\lceil\log n\rceil+\lceil\log (k+1)\rceil+7}$, no are sent to the environment and the computation halts. Thus, the answer of the system is negative. 


\subsection{Some formal details}

Family $\Pi=\{\Pi(\langle n, k\rangle) \mid n, k \in \mathbb{N}\}$ is polynomially uniform by Turing machines, because the rules of a system $\Pi(\langle n, k\rangle)$ of the family are defined recursively from the values $n$ and $k$. Besides, the necessary resources for defining each such system are of polynomial order.

- size of the alphabet: $\left(\left(2 n^{2}+3 n+8\right) \cdot\lceil\log (k+1)\rceil+n \cdot\lceil\log (k+1)\rceil^{2}\right.$ $+12 n+8\lceil\log n\rceil+46) / 2 \in O\left(n^{2} \cdot\lceil\log k\rceil+n \cdot\lceil\log k\rceil^{2}\right)$;

- initial number of membranes: $2 \in O(1)$;

- initial number of objects: $n \cdot\lceil\log (k+1)\rceil+n+10 \in O(n \cdot\lceil\log k\rceil)$;

- number of rules: $\left(\left(2 n^{2}-3 n+8\right) \cdot\lceil\log (k+1)\rceil+3 n \cdot\lceil\log (k+1)\rceil^{2}\right.$ $+8\lceil\log n\rceil+20 n+40) / 2 \in O\left(n^{2} \cdot\lceil\log k\rceil+n \cdot\lceil\log k\rceil^{2}\right)$;

- maximum length of a rule: $3 \in O(1)$.

Hence, there exists a deterministic Turing machine that builds the system $\Pi(\langle n, k\rangle)$ in a polynomial time with respect to $n$ and $k$.

According to the above mentioned computation process, it is clear that $\mathrm{P}$ system $\Pi(\langle n, k\rangle)$ with input multiset $\operatorname{cod}(u)$ always halts and sends to the environment object yes (at step $n+\lceil\log n\rceil+\lceil\log (k+1)\rceil+6$ ) or object no (at step $n+\lceil\log n\rceil+\lceil\log (k+1)\rceil+7)$. Therefore, there exists a polynomial bound for the number of steps of the computation.

Hence, the family $\Pi$ of recognizer $P$ systems with symport/antiport rules and membrane division solves the subset Sum problem in polynomial time according to Definition 3. So, the following result is obtained.

Theorem 1. SubsetSum $\in \mathbf{P M C}_{\mathbf{C D}_{e} \mathbf{C}(3)}$.

Corrolary 1. $\mathbf{N P} \cup \mathrm{co}-\mathrm{NP} \subseteq \mathbf{P M C}_{\mathbf{C D}_{e} \mathrm{C}(3)}$.

Proof 1. It suffices to make the following observations: the subset Sum problem is NP-complete, SubsetSum $\in \mathbf{P M C}_{\mathbf{C D}_{e} \mathbf{C}(3)}$ and the class $\mathbf{P M C}_{\mathbf{C D}_{e} \mathbf{C}(3)}$ is closed under polynomial time reduction, and is also closed under complement. $\square$

\section{Solving the $\mathrm{QSAT}$ problem by using $\mathrm{CD}_{n e} \mathbf{C}(3)$}

In this Section, we provide a (uniform) polynomial time solution to the QSAT problem (quantified satisfiability problem), a well-known PSPACE-complete problem Papadimitriou (1994), by a family of P systems with division rules for non-elementary membranes and symport/antiport of length at most 3.

Given a Boolean formula $\varphi\left(x_{1}, \ldots, x_{n}\right)$ in conjunctive normal form, with Boolean variables $x_{1}, \ldots, x_{n}$, the sentence $\varphi^{*}=\exists x_{1} \forall x_{2} \ldots Q_{n} x_{n} \varphi\left(x_{1}, \ldots, x_{n}\right)$ (where $Q_{n}$ is $\exists$ if $n$ is odd, and $Q_{n}$ is $\forall$ otherwise) is said to be the (existential) fully quantified formula associated with $\varphi\left(x_{1}, \ldots, x_{n}\right)$. We say that $\varphi^{*}$ is satisfiable if there exists a truth assignment, $\sigma$, over $\{i: 1 \leq i \leq n \wedge i$ odd $\}$ such that each extension, $\sigma^{*}$, of $\sigma$ over $\{1, \ldots, n\}$ verifies $\sigma^{*}\left(\varphi\left(x_{1}, \ldots, x_{n}\right)\right)=1$.

The QSAT problem is the following one: Given the (existential) fully quantified formula $\varphi^{*}$ associated with a Boolean formula $\varphi\left(x_{1}\right.$, ..., $x_{n}$ ) in conjunctive normal form, determine whether or not $\varphi^{*}$ is satisfiable.

The solution proposed follows a brute force algorithm in the framework of recognizer P systems with symport/antiport rules and membrane division, and it consists of the following phases:

- Generation phase: using membrane division for non-elementary membranes, all truth assignments for the variables associated with the Boolean formula are produced.

- Checking phase: checking whether or not the formula $\varphi\left(x_{1}, \ldots, x_{n}\right)$ is satisfied.

- Quantifier phase: checking whether the whole formula $\varphi^{*}$ with quantifiers is satisfied.

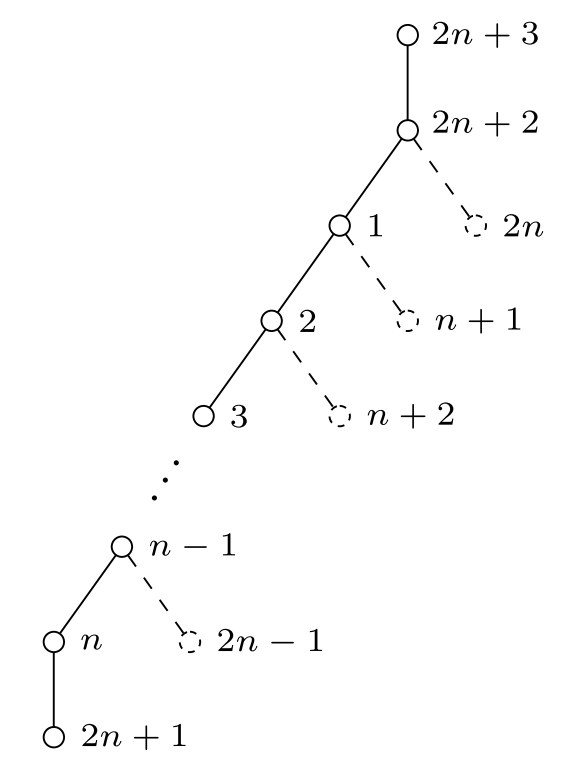

Fig. 1. The initial membrane structure of the P system.

- Output phase: the system sends to the environment the right answer according to the results of the previous phase.

We define a family $\Pi=\{\Pi(t) \mid t \in \mathbb{N}\}$ of recognizer P systems from $\mathbf{C D}_{n e} \mathbf{C}(3)$ such that each system $\Pi(t)$ will process all instances of the QSAT problem with $n$ variables and $m$ clauses, where $t=\langle m$, $n\rangle$, provided that the appropriate input multiset is supplied to the system.

For each $(m, n) \in \mathbb{N} \times \mathbb{N}$, we consider the recognizer P system from $\mathbf{C D}_{n e} \mathbf{C}(3)$,

$\Pi(\langle m, n\rangle)=\left(\Gamma, \mathcal{E}, \Sigma, \mu, \mathcal{M}_{1}, \ldots, \mathcal{M}_{2 n+3}, \mathcal{R}_{1}, \ldots, \mathcal{R}_{2 n+3}, i_{\text {in }}, i_{\text {out }}\right)$, defined as follows:

- $\Sigma=\left\{x_{i, j}, \bar{x}_{i, j} \mid 1 \leq i \leq n, 1 \leq j \leq m\right\}$.

- $\mathcal{E}=\left\{d_{i}, g_{i} \mid 0 \leq i \leq n^{2}+3 n+2 m+3-k\right\} \cup\left\{g_{n^{2}+3 n+2 m+4-k}\right\}$

(where $k=\left\lfloor\frac{n}{2}\right\rfloor$ is the number of universal quantifiers in $\varphi^{*}$ ).

- $\Gamma=\Sigma \cup \mathcal{E} \cup\left\{a_{i}, b_{i}, t_{i}, f_{i} \mid 1 \leq i \leq n\right\} \cup\left\{E_{i} \mid 0 \leq i \leq m+1\right\} \cup$

$\{t, y e s$, no $\}$.

- $\mu=\left[\left[\left[\left[\ldots\left[\left[[]_{2 n+1}\right]_{n}[]_{2 n-1}\right]_{n-1} \ldots\right]_{2}[]_{n+1}\right]_{1}[]_{2 n}\right]_{2 n+2}\right]_{2 n+3}$ (the root is the membrane with label $2 n+3$ whose child is labeled by $2 n+2$; this membrane has two children labeled by $2 n$ and 1 , respectively; each membrane with label $i, 1 \leq i \leq n-1$, contains a non-elementary membrane with label $i+1$ and an elementary membrane with label $n+i$, see Fig. 1).

- $\mathcal{M}_{1}=\left\{a_{1}\right\}, \mathcal{M}_{n+i}=\left\{b_{i}\right\}, 1 \leq i \leq n, \mathcal{M}_{2 n+2}=\mathcal{M}_{i}=\emptyset, 2 \leq i \leq n$.

- $\mathcal{M}_{2 n+1}=\left\{t, a_{2}, \ldots, a_{n}, E_{0}, E_{1}, \ldots, E_{m+1}\right\}, \quad \mathcal{M}_{2 n+3}=$ $\left\{d_{0}, g_{0}\right.$, yes, no $\}$.

- $i_{\text {in }}=2 n+1$ istheinputmembrane, and $i_{\text {out }}=0$ istheoutputregion .

- The sets of rules are defined below:

- Rules in $\mathcal{R}_{i}(1 \leq i \leq n): r_{1, i} \equiv\left[a_{i}\right]_{i} \rightarrow\left[t_{i}\right]_{i}\left[f_{i}\right]_{i}$.

- Rules in $\mathcal{R}_{i}(2 \leq i \leq n): r_{2, i j} \equiv\left(t_{j}\right.$, in $)$ and $r_{3, i j} \equiv\left(f_{j}, i n\right), 1 \leq j \leq i-1$.

- Rules in $\mathcal{R}_{i}(3 \leq i \leq n): r_{4, i j} \equiv\left(a_{j}\right.$,out $), 2 \leq j \leq i-1$.

- Rules in $\mathcal{R}_{2 n+1}$ : $r_{5, i} \equiv\left(a_{i+1}\right.$, out $; t_{i}$, in $), 1 \leq i \leq n-1$.

$r_{6, i} \equiv\left(a_{i+1}\right.$, out $; f_{i}$, in $), 1 \leq i \leq n-1$.

$r_{7} \equiv\left(E_{0} E_{1}\right.$, out $; t_{n}$, in $)$.

$r_{8} \equiv\left(E_{0} E_{1}\right.$, out $; f_{n}$, in $)$.

$r_{9, i, j} \equiv\left(t_{i} x_{i, j}\right.$, out $; E_{j}$, in $), 1 \leq i \leq n, 1 \leq j \leq m$.

$r_{10, i, j} \equiv\left(f_{i} \bar{x}_{i, j}\right.$, out $E_{j}$, in $), 1 \leq i \leq n, 1 \leq j \leq m$.

$r_{11, i, j} \equiv\left(E_{j+1}\right.$, out $; t_{i} x_{i, j}$, in $), 1 \leq i \leq n, 1 \leq j \leq m$. 
$r_{12, i, j} \equiv\left(E_{j+1}\right.$, out $; f_{i} \bar{x}_{i, j}$, in $), 1 \leq i \leq n, 1 \leq j \leq m$.

$r_{13} \equiv\left(t\right.$, out $; E_{m+1}$, in $)$.

- Rules in $\mathcal{R}_{n}: r_{14} \equiv(t$, out $)$.

- If $Q_{i+1}=\forall(1 \leq i \leq n-1)$ :

( $\star$ Rules in $\mathcal{R}_{n+i}: r_{15, i} \equiv\left(b_{i}\right.$, out $; t$, in $)$.

( $\star$ Rules in $\mathcal{R}_{i}: r_{16, i} \equiv\left(b_{i} t\right.$, out $)$.

- If $Q_{i+1}=\exists(1 \leq i \leq n-1)$ :

( $\star$ ) Rules in $\mathcal{R}_{n+i}: r_{17, i} \equiv(t$, in $)$ and $r_{18, i} \equiv\left(b_{i} t\right.$, out $)$.

( $\star$ Rules in $\mathcal{R}_{i}: r_{19, i} \equiv\left(b_{i} t\right.$, out $)$.

- Rules in $\mathcal{R}_{2 n}: r_{20} \equiv(t$, in $)$ and $r_{21} \equiv\left(b_{n} t\right.$, out $)$.

- Rules in $\mathcal{R}_{2 n+2}: r_{22} \equiv\left(b_{n} t\right.$, out $)$.

- Rules in $\mathcal{R}_{2 n+3}$ :

$r_{23} \equiv\left(d_{n^{2}+3 n+2 m+3-k}\right.$ tyes, out $)$.

$r_{24, i} \equiv\left(d_{i}\right.$, out $; d_{i+1}$, in $), 0 \leq i \leq n^{2}+3 n+2 m+2-k$.

$r_{25, i} \equiv\left(g_{i}\right.$, out $; g_{i+1}$, in $), 0 \leq i \leq n^{2}+3 n+2 m+3-k$.

$r_{26} \equiv\left(d_{n^{2}+3 n+2 m+3-k} g_{n^{2}+3 n+2 m+4-k} n o\right.$, out $)$.

\subsection{An overview of the computation}

In what follows, we informally describe how P system $\Pi\left(s\left(\varphi^{*}\right)\right)$ with input multiset $\operatorname{cod}\left(\varphi^{*}\right)$ works. Let us recall that $k$ denotes the number of universal quantifiers in $\varphi^{*}$, that is, $k=\left\lfloor\frac{n}{2}\right\rfloor$.

At the initial configuration, we have object $a_{1}$ in membrane 1 , object $b_{i}(1 \leq i \leq n)$ in membrane $n+i$, objects $a_{2}, \ldots, a_{n}, E_{1}, \ldots, E_{m+1}$, $t, \operatorname{cod}\left(\varphi^{*}\right)$ in membrane $2 n+1$, objects $g_{0}$, yes, no in membrane $2 n+3$.

Let us start with the generation phase. This phase has two parallel processes. On the one hand, the system assigns truthassignments to all variables $x_{i}(1 \leq i \leq n)$. When generation phase completes, we produce $2^{n}$ copies of membrane $2 n+1$, each of them contains a different truth assignments of the variable set $\left\{x_{1}, \ldots\right.$, $\left.x_{n}\right\}$ associated with the Boolean formula. On the other hand, the counter objects $d_{i}, g_{i}$ in membrane $2 n+3$ grow their subscripts by using rules $r_{26, i}, r_{27, i}$. We describe the process of generation phase as follows.

With the appearance of object $a_{i}$ in each membrane $i$ at the same step, the system starts to assign truth-assignment of variable $x_{i}$ $(1 \leq i \leq n-1)$. Specifically, by using rule $r_{1, i}$ in all membranes $i$, each non-elementary membrane $i$ is divided into two copies with the same label, which contain objects $t_{i}$ (representing the truth value true) and $f_{i}$ (representing the truth value false), respectively. Note that all the membranes and objects placed inside the membrane $i$ are replicated in the new copies. At next steps, all object $t_{i}\left(f_{i}\right.$, respectively) in membranes with label $i$ will be sent to membranes $n$ by using rules $r_{2, j, i}\left(r_{3, j, i}\right.$, respectively) $(i+1 \leq j \leq n)$ one by one. When object $t_{i}\left(f_{i}\right.$, respectively) appears in each membrane $n$ at the same step, rule $r_{5, i}\left(r_{6, i}\right.$, respectively) is enabled and applied, object $a_{i+1}$ in membrane $2 n+1$ is exchanged with object $t_{i}\left(f_{i}\right.$, respectively) in membrane $n$. When object $a_{i+1}$ appears in membranes $n$, by using rules $r_{4, j, i+1}(i+2 \leq j \leq n)$ one by one, object $a_{i+1}$ will be presented in each membrane $i+1$ at the same step. Similar to the case of variable $x_{i}$, the system continues to assign a truth-assignment to variable $x_{i+1}$. It is easy to see that the process of assigning truth-assignment of variable $x_{i}(1 \leq i \leq n-1)$ takes $2 n-2 i+1$ steps. With the appearance of object $a_{n}$ in membranes $n$ at the same step, the system starts to assign truth-assignment of variable $x_{n}$. By using rule $r_{1, n}$, each non-elementary membrane $n$ is divided into two copies at the same step, which contain objects $t_{n}$ (representing the truth value true) and $f_{n}$ (representing the truth value false), respectively. At the next step, rule $r_{7}\left(r_{8}\right.$, respectively) is enabled and applied, objects $E_{0}, E_{1}$ in membranes $2 n+1$ are exchanged with object $t_{n}$ $\left(f_{n}\right.$, respectively) in membranes $n$. The process of assigning a truthassignment to variable $x_{n}$ takes two steps. Hence, the generation phase takes $n^{2}+1$ steps. The membrane structure of the system at that moment when the generation phase completes is shown in Fig. 2.
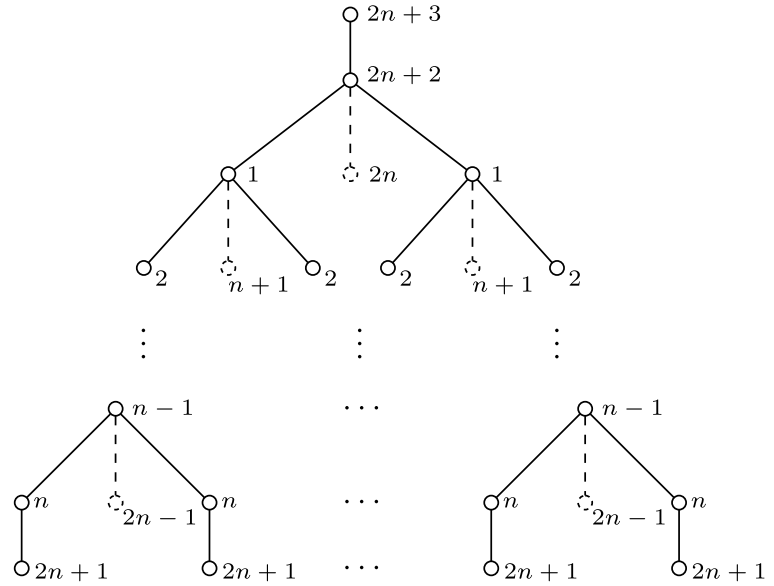

Fig. 2. The membrane structure of the system when the generation phase completes. Numbers at nodes indicate labels of membranes.

The checking phase takes $2 m$ steps and consists of $m$ loops (each loop takes 2 steps). In parallel with checking whether there is a truth assignment that makes the formula $\varphi$ evaluate to be true, the counter objects $d_{i}, g_{i}$ also grow their subscripts by one for each step in membrane $2 n+3$.

At the first step of the $j$-th loop $(1 \leq j \leq m)$ of checking phase, objects $t_{i}, x_{i, j}\left(f_{i}, \bar{x}_{i, j}\right.$, respectively) in membrane $2 n+1$ are exchanged with object $E_{j}$ in membrane $n$ by using rule $r_{9, i, j}\left(r_{10, i, j}\right.$, respectively), in case membrane $2 n+3$ encodes a truth assignment making clauses $C_{1}, \ldots, C_{j}$ true. Note that for any membrane $2 n+1$, which contains a truth assignment that does not make the clause $C_{j}$ true, the computation in that membrane stops at the time when $r_{9, i, j}$ or $r_{10, i, j}$ is applied. At the second step of the $j$-th loop $(1 \leq j \leq m)$ of checking phase, with the appearance of objects $t_{i}, x_{i, j}\left(f_{i}, \bar{x}_{i, j}\right.$, respectively) in membrane $n$, by using rule $r_{11, i, j}\left(r_{12, i, j}\right.$, respectively), object $E_{j+1}$ in membrane $2 n+1$ is exchanged with the objects $t_{i}, x_{i, j}\left(f_{i}, \bar{x}_{i, j}\right.$, respectively) in membrane $n$.

After $n^{2}+2 m+1$ steps, we have checked whether or not formula $\varphi$ is satisfied by the corresponding truth assignment. For each clause $C_{j}$ which is satisfied, the subscript $j$ of $E$ is increased by one; hence object $E_{m+1}$ will appear in membrane $n$ if and only if its lower neighbor membrane $2 n+1$ encodes the truth assignment that satisfies all clauses. At step $n^{2}+2 m+2$, if membrane $n$ contains $E_{m+1}$ then by using rule $r_{13}$, object $t$ in membrane $2 n+1$ is exchanged with object $E_{m+1}$ in membrane $n$. At the next step, if object $t$ appears in membrane $n$, by applying rule $r_{14}$, then it will be sent to membrane $n-1$.

The quantifier phase starts at the $\left(n^{2}+2 m+4\right)$-th step. In membrane $2 n+3$ the counter objects $d_{i}, g_{i}$ grow their subscripts by one for each step. A membrane with label $i$ corresponds to the quantifier $Q_{j}$, where $1 \leq i \leq n-1, j=i+1$, and a membrane with label $2 n+2$ corresponds to the quantifier $Q_{1}$. If $Q_{j}=\forall$, object $t$ is passed to the upper level only if it comes from both lower level membranes, that is, the respective clauses are satisfied for both truth values of $x_{j}$. If $Q_{j}=\exists$, then a single object $t$ coming from lower level is enough. In what follows, we describe how the system simulates quantifiers $\forall$ and $\exists$.

For quantifier $Q_{i+1}=\forall(1 \leq i \leq n-1$ and $i$ is odd $)$, one copy of object $t$ can be sent to the upper level membrane if and only if there are two copies of object $t$ in a membrane $i$, where each lower level membrane provides one copy of object $t$. Specifically, by using rule $r_{15, i}$, object $b_{i}$ in membranes $n+i$ is exchanged with object $t$ in their upper level membranes $i$. Note that there is one copy of object $b_{i}$ in a membrane $n+i$, thus, only one copy of object $t$ is sent to membrane $n+i$ from a membrane $i$. At the next step, objects $b_{i}, t$ in membranes $i$ are sent to their upper level membranes by applying rule $r_{16, i}$. 
In case $Q_{i+1}=\exists\left(1 \leq i \leq n-1\right.$ and $i$ is even), by using the rule $r_{17, i}$ in all membranes $n+i$, all copies of object $t$ will be sent to their lower level membranes $n+i$. At the next step, objects $b_{i}, t$ are sent to membrane $i$ by using rule $r_{18, i}$. If there are two copies of object $t$ in membrane $n+i$, then only one copy of object $t$ is sent to membrane $i$ because there is only one copy of object $b_{i}$ in a membrane $n+i$. At the next step, objects $b_{i}, t$ in membranes $i$ are sent to their upper level membranes by using rule $r_{19, i}$. As $Q_{1}=\exists$, by using the rules $r_{20}$, $r_{21}, r_{22}$ one by one, one copy of object $t$ is sent to the membrane $2 n+3$.

Hence, the simulation of all universal quantifiers takes $2 k$ steps, and the simulation of all existential quantifiers takes $3(n-k)$ steps (the number of such quantifiers is $n-k$ ). Thus, the quantifier phase takes $3 n-k$ steps.

The output phase starts at the $\left(n^{2}+3 n+2 m+4-k\right)$-th step, and it takes 1 step (affirmative answer) or 2 steps (negative answer). Let us recall that membrane $2 n+3$ at configuration $\mathcal{C}_{n^{2}+3 n+2 m+3-k}$ contains objects $d_{n^{2}+3 n+2 m+3-k}, g_{n^{2}+3 n+2 m+3-k}$, yes and no. There are two cases.

- Affirmative answer: if object $t$ appears in membrane $2 n+3$ at configuration $\mathcal{C}_{n^{2}+3 n+2 m+3-k}$, then rules $r_{23}$ and $r_{25, n^{2}+3 n+2 m+3-k}$ are applicable and objects $d_{n^{2}+3 n+2 m+3-k}, t$ and yes are sent to the environment. Membrane $2 n+3$ at configuration $\mathcal{C}_{n^{2}+3 n+2 m+4-k}$ only contains objects $g_{n^{2}+3 n+2 m+4-k}$ and no. Therefore, the computation halts.

- Negative answer: if object $t$ does not appear in membrane $2 n+3$ at configuration $\mathcal{C}_{n^{2}+3 n+2 m+3-k}$, then only rule $r_{25, n^{2}+3 n+2 m+3-k}$ is applicable. Thus, that membrane at configuration $\mathcal{C}_{n^{2}+3 n+2 m+4-k}$ contains objects yes, no, $g_{n^{2}+3 n+2 m+4-k}, d_{n^{2}+3 n+2 m+3-k}$. At the next step, by using rule $r_{26}$, objects $g_{n^{2}+3 n+2 m+4-k}, d_{n^{2}+3 n+2 m+3-k}$ and no are sent to the environment and membrane $2 n+3$ at configuration $\mathcal{C}_{n^{2}+3 n+2 m+5-k}$ only contains object yes, so it is a halting configuration.

\subsection{Some formal details}

In order to show that the family $\Pi=\{\Pi(\langle m, n\rangle) \mid m, n \in \mathbb{N}\}$ defined above is polynomially uniform by Turing machines we need to prove that $\Pi(\langle m, n\rangle)$ is built in polynomial time with respect to the size parameter $m, n$ of instances of the QSAT problem (recalling that $k \leq n$ ).

It is easy to show that the rules of a system $\Pi(\langle m, n\rangle)$ of the family are defined recursively from the values $m, n$, and the necessary resources to construct $\Pi(\langle m, n\rangle)$ are as follows:

- size of the alphabet: $2 n^{2}+2 n m+10 n+5 m-2 k+12 \in O\left(n^{2}+n m\right)$;

- initial number of membranes: $2 n+3 \in O(n)$;

- initial number of objects: $2 n+m+7 \in O(n+m)$;

- number of rules: $\left(7 n^{2}+8 n m+23 n+8 m-4 k+20\right) / 2 \in O\left(n^{2}+n m\right)$;

- maximum length of a rule: $3 \in O(1)$.

Thus, there exists a deterministic Turing machine that builds the system $\Pi(\langle m, n\rangle)$ in a polynomial time with respect to $m$ and $n$.

By the above checking of the computation process, we can prove that the P system $\Pi(\langle m, n\rangle)$ with input multiset $\operatorname{cod}\left(\varphi^{*}\right)$ always halts and sends to the environment object yes or no in the last step, that is, at step $n^{2}+3 n+2 m+4-k$, object yes is sent to the environment and the system halts; object no is sent to the environment at step $n^{2}+3 n+2 m+5-k$ and the system halts. Therefore, there exists a polynomial bound for the number of steps of the computation.

Hence, the family $\boldsymbol{\Pi}$ of recognizer $\mathrm{P}$ systems with symport/antiport rules and membrane division solves the QSAT problem in polynomial time according to Definition 3. So, the following result is obtained.

\section{Theorem 2. QSAT $\in \mathbf{P M C}_{\mathbf{C D}_{n e} \mathbf{C}(3)}$.}

Since the complexity class $\mathbf{P} \mathbf{M C} \mathbf{C D}_{n e} \mathbf{C}(3)$ is closed under polynomial time reductions, we have the following result.

\section{Corrolary 2. $\quad$ PSPACE $\subseteq \mathbf{P M C}_{\mathbf{C D}_{n e} \mathbf{C}(3)}$.}

\section{Conclusions and discussion}

The computational efficiency of different computing devices in membrane computing has been established. With regard to celllike computing devices, efficient solutions to hard problems have been given in the framework of P systems with active membranes that use evolution, send-in, send-out, dissolution and division rules. Specifically, polynomial time solutions to the SAT problem PérezJiménez et al. (2003) (in a uniform way by using electrical charges) and the QSAT problem Alhazov and Pérez-Jiménez (2007) (in a semi-uniform way, without electrical charges and allowing division for non-elementary membranes) have been proposed. With regard to tissue-like computing devices, an efficient (uniform) solution to the HAM-CYCLE problem by a family of tissue P systems with cell division and symport/antiport rules of length at most 2, has been given Porreca et al. (2012).

In this work, the computational efficiency of cell-like polarizationless P systems with symport/antiport rules and membrane division has been investigated. Specifically, a (uniform) linear time solution to the NP-complete problem subset Sum by using division rules for elementary membranes and communication rules of length at most 3 , has been given. We further proved that such $P$ system can efficiently solve the PSPACE-complete problem QSAT problem, in a uniform way, when division rules for non-elementary membranes are allowed. It is worth noting that the solution to QSAT given in the paper can be adapted to a uniform solution bearing in mind that for (existential) fully quantified formula associated with $\varphi\left(x_{1}, \ldots, x_{n}\right)$, the number of universal quantifiers is $\left\lfloor\frac{n}{2}\right\rfloor$.

We propose some open problems related to the role of communication rules in cell-like $P$ systems with membrane division from a computational complexity point of view.

(a) In the solution to QSAT proposed in this paper, division rules for non-elementary membranes have been considered. Is it possible to provide an efficient solution to QSAT by using only division rules for elementary membranes?

(b) The P systems constructed in Section 4 and in Section 5 have both symport rules and antiport rules. What about the computational efficiency of $P$ systems with membrane division that use only either symport or antiport rules?

(c) It is known that $\mathbf{N P} \cup \mathbf{c o}-\mathbf{N P} \subseteq \mathbf{P M C}_{\mathbf{T D C}(2)}$ Porreca et al. (2012). What about the computational efficiency of $\mathbf{C D}_{e} \mathbf{C}(2)$ ?

(d) The environment is not relevant for tissue P systems with symport/antiport rules and cell division from a complexity point of view Pérez-Jiménez et al. (2013). What about the efficiency of recognizer P systems from $\mathbf{C D C}(k)$ when the alphabet of the environment is an empty set?

P systems capture the inherent degree of freedom present in biological systems through the non-determinism. With the inclusion of this ingredient, P systems are able to solve hard problems in an "efficient" way at the theoretical level by trading time for space. New boundaries between tractability and NP-hardness, in terms of syntactical ingredients of P systems, provide new tools to tackle the $\mathbf{P}$ versus NP problem.

Different simulators for $\mathrm{P}$ systems running on conventional computers have been developed during the last decade. The addition of parallel computing techniques, like those based on GPU, is accelerating these simulators. With the aid of P systems simulators, researchers aim to solve larger instances of NP hard problems than 
the best ones provided so far, running on electronic computers. However, we would like to stress that such simulators are not real implementation of P systems. Obviously, an efficient real implementation of $\mathrm{P}$ systems would provide a "constructive" proof of the result $\mathbf{P}=\mathbf{N P}$, that is, similarly to what would happen with a practical implementation of non-deterministic Turing machines.

\section{Acknowledgements}

The work of B. Song and L. Pan was supported by National Natural Science Foundation of China (61033003, 91130034, and 61320106005), Ph.D. Programs Foundation of Ministry of Education of China (2012014213008), and Natural Science Foundation of Hubei Province (2011CDA027). The work of M.J. Pérez-Jiménez was supported by "Ministerio de Economía y Competitividad" of Spain (TIN2012-37434), cofunded by FEDER funds.

\section{References}

Alhazov, A., Freund, R., 2005. P systems with one membrane and symport/antiport rules of five symbols are computationally complete. In: Proceedings of Third Brainstorming Week On Membrane Computing, Sevilla, Spain, pp. 19-28.

Alhazov, A., Rogozhin, Yu., 2006. Towards a characterization of P systems with minimal symport/antiport and two membranes. In: Vol. 4361 of Lecture Notes in Computer Science. Springer, Berlin/Heidelberg, pp. 135-153.

Alhazov, A., Pérez-Jiménez, M.J., 2007. Uniform solution of QSAT using polarizationless active membranes. In: Vol. 4664 of Lecture Notes in Computer Science. Springer, Berlin/Heidelberg, pp. 122-133.

Bernardini, F., Gheorghe, M., 2003. On the power of minimal symport/antiport. In: Proceedings of the 3rd Workshop on Membrane Computing, Tarragona, pp. 72-83.

Ciobanu, G., Pan, L., Păun, Gh., Pérez-Jiménez, M.J., 2007. P systems with minimal parallelism. Theor. Comput. Sci. 378, 117-130.

Díaz-Pernil, D. Gutiérrez-Naranjo, M.A., Pérez-Jiménez, M.J., Riscos-Núñez, A., 2007. A linear solution for Subset Sum problem with tissue P systems with cell division. In: Vol. 4527 of Lecture Notes in Computer Science. Springer, Berlin/Heidelberg, pp. $170-179$.

Frisco, P., 2009. Computing with Cells: Advances in Membrane Computing. Oxford University Press, Oxford.
Gutiérrez-Naranjo, M.A., Pérez-Jiménez, M.J., Romero-Campero, F.J., 2005. A linear solution of subset sum problem by using membrane creation. In: Vol. 3561 of Lecture Notes in Computer Science. Springer, Berlin/Heidelberg, pp. 258-267.

Ionescu, M., Păun, Gh., Yokomori, T., 2006. Spiking neural P systems. Fund. Inform. $71(2-3), 279-308$

Martín-Vide, C., Pazos, J., Păun, Gh., Rodriguez-Paton, A., 2003. Tissue P systems. Theor. Comput. Sci. 296 (2), 295-326.

Papadimitriou, C.H., 1994. Computational Complexity. Addison-Wesley, Reading Mass.

Păun, A., 2000. On P systems with active membranes. In: Proceedings of the Second International Conference on Unconventional Models of Computation, UMC'2K, Brussels, Belgium, pp. 187-201.

Păun, A., Păun, Gh., 2002. The power of communication: P systems with symport/antiport. New Gener. Comput. 20 (3), 295-305.

Păun, Gh., 2000. Computing with membranes. J. Comput. Syst. Sci. 61 (1), 108-143.

Păun, Gh., 2001. P systems with active membranes: attacking NP-complete problems. J. Auto. Lang. Comb. 6, 75-90.

Păun, Gh., Pazos, J., Pérez-Jiménez, M.J., Rodríguez-Patón, A., 2005. Symport/antiport P systems with three objects are universal. Fund. Inform. 64, 1-4.

Păun, Gh., Rozenberg, G., Salomaa, A., 2010. The Oxford Handbook of Membrane Computing. Oxford University Press, Oxford.

Pérez-Jiménez, M.J., Romero-Jiménez, A., Sancho-Caparrini, F., 2003. Complexity classes in models of cellular computing with membranes. Nat. Comput. 2 (3), 265-285.

Pérez-Jiménez, M.J., Riscos-Núñez, A., 2004. A linear time solution to the Knapsack problem using P systems with active membranes. In: Vol. 2933 of Lecture Notes in Computer Science. Springer, Berlin/Heidelberg, pp. 250-268.

Pérez-Jiménez, M.J., Riscos-Núñez, A., 2005. Solving the Subset-Sum problem by active membranes. New Gener. Comput 23, 367-384.

Pérez-Jiménez, M.J., 2005. An approach to computational complexity in membrane computing. In: Vol. 3365 of Lecture Notes in Computer Science. Springer Berlin/Heidelberg, pp. 85-109.

Pérez-Jiménez, M.J., Romero-Jiménez, A., Sancho-Caparrini, F., 2006. A polynomia complexity class in P systems using membrane division. J. Auto. Lang. Comb. 11 (4), 423-434.

Pérez-Jiménez, M.J., Riscos-Núñez, A., Rius-Font, M., Romero-Campero, F.J., 2013. A polynomial alternative to unbounded environment for tissue P systems with cell division. Int. J. Comput. Math. 90 (4), 760-775.

Porreca, A.E., Murphy, N., Pérez-Jiménez, M., 2012. An optimal frontier of the efficiency of tissue P systems with cell division. In: Proceedings of the Tenth Brainstorming Week on Membrane Computing, Volume II, Sevilla, Spain, pp. 141-166.

Rozenberg, G., Salomaa, A., 1997. Handbook of Formal Languages, vol. 3. SpringerVerlag, Berlin. 